\title{
Multiscale mathematical modeling of aqueous humor dynamics: Applications for precision care in glaucoma
}

Lang, Matthew ${ }^{1}$; Harris, Alon ${ }^{1}$; Verticchio Vercellin, Alice Chandra ${ }^{2,3}$; Mathew, Sunu'; Guidoboni, Giovanna ${ }^{4}$

1. Ophthalmology, Indiana University School of Medicine, Indianapolis, IN, United States. 2. Ophthalmology, University of Pavia, Italy, 3. IRCCS - Fondazione Bietti, Italy, 4. University of Missouri, Columbia, MO, United States

\section{Background and Hypothesis:}

Reduction of intraocular pressure (IOP) resulting from overproduction or impaired outflow of aqueous humor is, to date, the only approved medical treatment for the disease. IOP is determined by the balance between aqueous humor production at the ciliary body and drainage at the anterior chamber angle. A mathematical model describing aqueous humor $(\mathrm{AH})$ flow was employed in order to study the effects of glaucoma medications and risk factors on IOP.

\section{Experimental Design or Project Methods:}

IOP can be calculated as the unknown variable in the mathematical balance between $A H$ inflow $\left(\mathrm{J}_{\text {in }}\right)$ and outflow ( $\left.\mathrm{J}_{\text {out }}\right)$. Model simulations using MATLAB were used to calculate IOP in conditions of varied trabecular meshwork resistance $(R)$ over changes in episcleral venous pressure (EVP), uveosclearal outflow facility $\left(\mathrm{C}_{\mathrm{uv}}\right)$, and ciliary capillary blood pressure (cBP) in order to simulate the effects of EVP reducing medications, prostaglandin analogs, and systemic BP, respectively.

\section{Results:}

The simulated effects of EVP reducing medications and prostaglandin analogs led to IOP decrease of $13.12 \%, 5.51 \%$, and $2.88 \%$ for conditions of $R_{0}, 3 R_{0}$, and $6 R_{0}$, respectively, where $R_{0}$ is equal to the trabecular meshwork resistance when IOP $=$ EVP. The simulated effects of prostaglandin analogs resulted an IOP decrease of $13.73 \%, 18.34 \%$, and $19.83 \%$, for conditions of $R_{0}, 3 R_{0}$, and $6 R_{0}$, respectively. The simulated effects of increasing systemic BP resulted in an IOP increase of $49.88 \%, 58.51 \%$, and $60.71 \%$ for conditions of $R_{0}, 3 R_{0}$, and $6 R_{0}$, respectively.

\section{Conclusion and Potential Impact:}

The model simulations predict differential efficacy in IOP reducing medications in patients of varied $R$ as well as the differential impact of systemic BP on IOP in patients of varied $R$. This model has the potential to predict the IOP reducing effect of medications in an individual as well as the impact of risk factors such as systemic BP on IOP. 